\title{
Gene Expression Profile of THP-1 Cells Infected by R. prowazekii Suggests Host Response Signature Genes
}

\author{
Hong Ge*,1, Shuping Zhao ${ }^{2}$, Xing Lü̈ ${ }^{2}$ Eric Yao-Yu Chuang ${ }^{3,4}$ and Wei-Mei Ching ${ }^{1,5}$
}

\author{
${ }^{I}$ Viral and Rickettsial Diseases Department, Infectious Diseases Directorate, Naval Medical Research Center, Silver \\ Spring, Maryland 20910, USA \\ ${ }^{2}$ Microarray Laboratory, Radiation Oncology Sciences Program, National Cancer Institute, National Institutes of \\ Health, Gaithersburg, Maryland 20877, USA \\ ${ }^{3}$ Radiation Biology Branch, Center for Cancer Research, National Cancer Institute, National Institutes of Health, Be- \\ thesda, Maryland 20892, USA \\ ${ }^{4}$ Graduate Institute of Bioelectronics and Bioinformatics, Department of Electrical Engineering, Center for Genomic \\ Medicine, National Taiwan University, Taipei, Taiwan \\ ${ }^{5}$ Department of Preventive Medicine and Biometrics, Uniformed Services University of the Health Sciences, Bethesda, \\ Maryland 20814, USA
}

\begin{abstract}
Rickettsia prowazekii, a selective agent of category B, is the causative microorganism of epidemic typhus. The genome-wide profile of host response to $R$. prowazekii infection was studied in THP-1 cells by a human cDNA microarray. Approximately $131(1.71 \%)$ and $11(0.14 \%)$ genes out of 7,680 genes assessed were up-regulated or down-regulated upon infection with virulent $R$. prowazekii strain Breinl. These genes induced by $R$. prowazekii were diverse in function. Six genes [ENG (endoglin or CD105, cell surface glycoprotein), GADD45A, TNFAIP3, IGFBP3, POU3F4 (transcription), ELK3] were identified as commonly induced genes as their over expressions were observed throughout the entire time course studied. There were twenty-two genes [such as GADD45A, POU3F4, ENG, PPP1R14B (an enzyme), ELK3, CXCL1, IL1B, NFKB1A, etc.] that exhibited the high level of induction at more than one time point. Collectively, these discoveries may provide novel insights into mechanisms of rickettsial pathogenesis and might reveal potential therapeutic targets against rickettsial infection.
\end{abstract}

\section{INTRODUCTION}

Rickettsiae are obligate intracellular Gram-negative bacteria. Rickettsial infections can cause acute fever, headache, skin rash, eschars, and in severe cases failure of the cardiovascular system. $R$. prowazekii, the causative agent of epidemic typhus, caused millions of deaths in both World Wars [1] and has reemerged worldwide recently [2]. It has been listed as select agent by the Centers for Disease Control and Prevention in the United States.

The host-rickettsia interaction is likely to involve a complex interplay between host defense and bacterial evasion as seen in other bacterial infections [3]. Based on the genome sequence of $R$. prowazekii Madrid E [4], the comparative genome-wide analysis of $R$. prowazekii virulent and avirulent strains (Breinl vs. Madrid E) revealed potential virulence genes that may provide useful information on the pathogenesis of Rickettsia [5]. Adherence to the membrane of target cell is a first key step in the establishment of a rickettsial infection. The increased expressions of the receptors E-selectin, intercellular adhesion molecules 1 (ICAM-1) and vascular cell adhesion molecule 1 (VCAM-1) have been reported in human endothelial cells post rickettsial infection

*Address correspondence to this author at the Bldg. 503, Rm 3N66, VRDD, IDD, NMRC, Silver Spring, MD20910, USA; Tel: 301-319-7439; Fax: 301319-3015; E-mail: geh@nmrc.navy.mil.com
[6]. It is believed that after adherence, rickettsiae enter into host cells and then rapidly escape from phagocytic vacuoles before phagolysosomal fusion [7]. Within the cytoplasm, rickettsiae induce rearrangement of host actin filaments in order to facilitate their intracellular spread and host cell locomotion [8]. A recent study of $R$. conorii has shown that the parasite surface protein, RickA, can recruit and activate Arp2/3, which then induces actin polymerization of host cells [8]. However, there is not much known about the sequence of events and molecules involved in rickettsial infection. The expression profile of host response to $R$. prowazekii infection has not been well investigated. With the availabilities of the human genome sequence and cDNA microarray technology, study of expression patterns on a global level has now become possible.

The aim of this study was to explore the host response to $R$. prowazekii infection by determining the global transcriptional response of a human monocytic cell line (THP-1 cells) upon infection. Human THP-1 cells in culture were harvested at various times post infection with $R$. prowazekii. The transcriptional expressions of THP-1 cells were analyzed on microchips containing 7,680 human cDNAs. From data mining and confirmation by quantitative RT-PCR, a characteristic pattern of responsive genes was identified. These results may yield insights into the mechanism of the human defense response to $R$. prowazekii infection. 


\section{MATERIALS AND METHODOLOGY}

\section{Host Cell Culture and Exposure to $R$. prowazekii Virulent Strain Breinl}

THP-1 cells originally derived from an acute monocytic leukemia patient were purchased from ATCC. The cells were cultured in RPMI 1640 with 10\% FBS for several days until the cell density reached $1-2 \times 10^{7}$ cells in $162 \mathrm{~cm}^{2}$ flask at $37^{\circ} \mathrm{C}, 5 \% \mathrm{CO}_{2}$. R. prowazekii Breinl were inoculated into THP-1 cells with multiplicities of infection around 25:1. The cultures were rocked at room temperature for one hour after which medium was removed and fresh medium was added, then the culture was returned to $37^{\circ} \mathrm{C}, 5 \% \mathrm{CO}_{2}$.

\section{Total RNA Isolation}

The cultures were harvested at $1,4,8,18$, and 24 hours (T1, T4, T8, T24) post-infection. Total RNA was extracted immediately using TRIzol (Invitrogen, Carlsbad, CA) following the protocol provided by the manufacturer. RNA was further purified using the RNeasy Mini Kit according to the manufacturer's instructions (Qiagen, Valencia, CA) with an additional step of on-column DNase Digestion using RNasefree DNase (Qiagen, Valencia, CA) to completely remove
DNA. The yield, integrity, and purity of total RNA were determined by agarose gel electrophoresis and the ratio of O.D. 260/280.

\section{Probe Labeling, Microarray Hybridization, and Image Analysis}

The microarray slides used for this study contained 7,680 human cDNA clones (National Cancer Institute ROSP 8K Human Array) purchased from Research Genetics (Huntsville, AL). The cDNAs were spotted onto poly-Llysine-coated slides by a computerized OMNI Grid Arrayer (GeneMachines, San Carlos, CA). THP-1 cells at T1-T24 post-infection and un-infected cells at corresponding time points were collected. Equal amount of RNAs $(20-40 \mu \mathrm{g})$ from infected and uninfected cells were reverse transcribed and labeled with fluorescent $\mathrm{Cy} 3$ or Cy5 analogs of dCTP, respectively (Amersham, Piscataway, NJ). The labeled cDNAs were purified, concentrated, and hybridized onto microarray slides.

The microarrays were scanned at a $10-\mu \mathrm{m}$ resolution on a GenePix 4000A scanner (Axon Instruments, Inc., Foster City) to obtain maximal signal intensities with $<1 \%$ probe saturation. The Cy5- and the Cy3-labeled cDNA samples

Table 1. Primers and Probes Used in Real-Time PCR

Target Sequence ( $\left.5^{\prime}-3^{\prime}\right)$

\begin{tabular}{|c|c|}
\hline GADD45A & \\
\hline Forward..... & $\ldots \ldots \ldots \ldots$ ' TGT GAG TGA GTG CAG AAA GCA G 3' \\
\hline Reverse..... & ............... $5^{\prime}$ CСA CCT TAT CCA TCC TTT CGG T 3 , \\
\hline Probe........ & ....5' TCT GCT CTC CAG CCG AGA ATT CCT CCA A 3, \\
\hline PPP1R14B & \\
\hline Forward.... & $\ldots \ldots \ldots \ldots . . .5$ ' GGA AGG TCA CCC TCA AGT ATG A 3' \\
\hline Reverse.... & .............. 5' TCA TCC ACG TCA ATC TCC AGT T 3 , \\
\hline Probe...... & ......5' TGG GAT CTC CTC TTC CTG GCA GTC GTA 3' \\
\hline ENG & \\
\hline Forward.... & $\ldots \ldots \ldots \ldots . . .5$ ' CAA CAT GGA CAG CCT CTC TTT C 3 ' \\
\hline Reverse.... & $\ldots \ldots \ldots \ldots$, TGT CTA ACT GGA GCA GGA ACT C 3 ' \\
\hline Probe........ & ........5' CTC TAC CTC AGC CCA CAC TTC CTC CA 3' \\
\hline NFKB1A & \\
\hline Forward.. & $\ldots \ldots \ldots \ldots . . . . .5$ ' AGA GAG TGA GGA TGA GGA GAG 3' \\
\hline Reverse... & $\ldots . . . . . . . . . . .5$ ACA CAG TCA TCA TAG GGC AG 3' \\
\hline Probe........ & ....... $5^{\prime}$ TGT GAA CTC CGT GAA CTC TGA CTC TGT 3 , \\
\hline ILB1 & \\
\hline Forward.. & $\ldots \ldots \ldots \ldots . .5$ ' АTT CTC TTC AGC CAA TCT TCA TT 3 ' \\
\hline Reverse..... & ................ GCC ATC AGC TTC AAA GAA CA 3, \\
\hline Probe........ & ...5' TCA TCC TCA TTG CCA CTG TAA TAA GCC A 3 ' \\
\hline CXCL1 & \\
\hline Forward... & $\ldots \ldots \ldots \ldots . . .5$ CAA AGT GTG AAC GTG AAG TCC C 3 ' \\
\hline Reverse.... & $\ldots \ldots \ldots$ ' TGT TCA GCA TCT TTT CGA TGA TTT 3' \\
\hline Probe........ & ........5' CTG CGC CCA AAC CGA AGT CAT AGC C 3' \\
\hline TNFAIP3 & \\
\hline Forward.... & ..........5' ACA GAC ACA CGC AAC TTT AA 3' \\
\hline Reverse..... & .....5' TTT GAT AAG ATT GTC CCA TTC ATC 3' \\
\hline Probe....... & .......5' CCG CTG GCA ACT GGA GTC TCT 3' \\
\hline
\end{tabular}


were scanned at $635 \mathrm{~nm}$ and $532 \mathrm{~nm}$, respectively. The resulting TIFF images were analyzed by Gene Pix Pro 4.0 software (Axon Instruments, Inc., Foster City). Microarray data were stored in the NIH microarray database. The ratios of intensities of the infected to control samples for all targets were determined after background subtraction. Raw intensity profiles were analyzed using the mAdb tools (National Center for Biotechnology Information, NIH). The data were further analyzed by using TreeView and Cluster [9]. Ingenuity Pathway Analysis (IPA) [10-11] was applied to the data as an additional method to further evaluate the functional significance of genes. IPA uses a curated database to construct different regulatory networks of imported genes. Each gene identified by IPA was mapped to its corresponding gene object in the Ingenuity Pathway Knowledge Base. This program uses its knowledge base to identify interactions between focus genes and other related genes in order to create networks. IPA produces a statistical score for each network according to the fit of the network to the set of focus genes. The score is the negative $\log$ of $\mathrm{p}$ and indicates the likelihood of the focus genes in the network due to random chance. A core of 3 means that there is a 1/1000 chance that the focus genes are in a network due to random chance. Therefore, the scores of 3 or higher have a $99.9 \%$ confidence of not being generated by random chance alone. This score was used as a cut-off point for identifying gene networks that are significantly regulated by $R$. prowazekii infection.

\section{Reverse Transcription and Real Time RT-PCR}

RT was carried out using MessageSensor ${ }^{\mathrm{TM}}$ RT Kit (Ambion), which was specifically designed for elevated sensitivity in real time PCR. Real time PCR primer pairs and probes for selected genes were designed using TaqMan Design 2.0 software (ABI, Weiterstadt, Germany) and are shown in Table 1. The sequences of exon and intron of those genes were searched and either primer or probe covered exon/exon junction to avoid the amplification of genomic DNA. The procedures for real time PCR were performed as described previously [5]. The probes were labeled with 6-carboxyfluorescein (FAM) and the new generation quencher (BHQ1) at the 5' and 3' ends, respectively, by Biosearch Technologies (Novato, CA). PCR cycling was conducted using a SmartCycler (Cepheid, Sunnyvale, CA) in a total volume of $25 \mu$ containing 1x TaqMan Universal PCR Master Mix (Applied Biosystems), $0.2 \mu \mathrm{M}$ of each primer, $0.1 \mu \mathrm{M}$ probe, and $1 \mu \mathrm{l}$ of diluted cDNA template. The probe and primers for $18 \mathrm{~S}$ rRNA gene purchased from Biosource International were included as an endogenous reference. The comparative $\mathrm{C}_{\mathrm{T}}$ (threshold cycles) method was applied using arithmetic formulas $\left(2_{T}^{-\Delta \Delta C}\right.$ ) (ABI system, AB, Piscataway, NJ). PCR reactions with no-cDNA templates were included as negative controls. Real time RT-PCR assays were performed in triplicate for each sample at every time point, and a mean value and standard deviation were calculated for the relative RNA expression levels.

\section{RESULTS}

\section{Hierarchical Clustering of Signature Genes and their Functional Categories}

To characterize the host gene response to $R$. prowazekii, a human cDNA microarray containing 7,680 genes was used. THP-1 cells infected with $R$. prowazekii were harvested at
T1-T24. Total RNA was extracted for cDNA synthesis. To exclude the possibility of cross hybridization of bacteria cDNA to human microarray slides, poly dT was used as primer for $\mathrm{Cy} 3$ or $\mathrm{Cy} 5$ incorporation reaction instead of random primers in all the labeling procedures since rickettsial mRNA lacks poly A at 3' end. The differences in signal intensities of co-hybridization experiments were compared for each spot between infected and uninfected control cells.

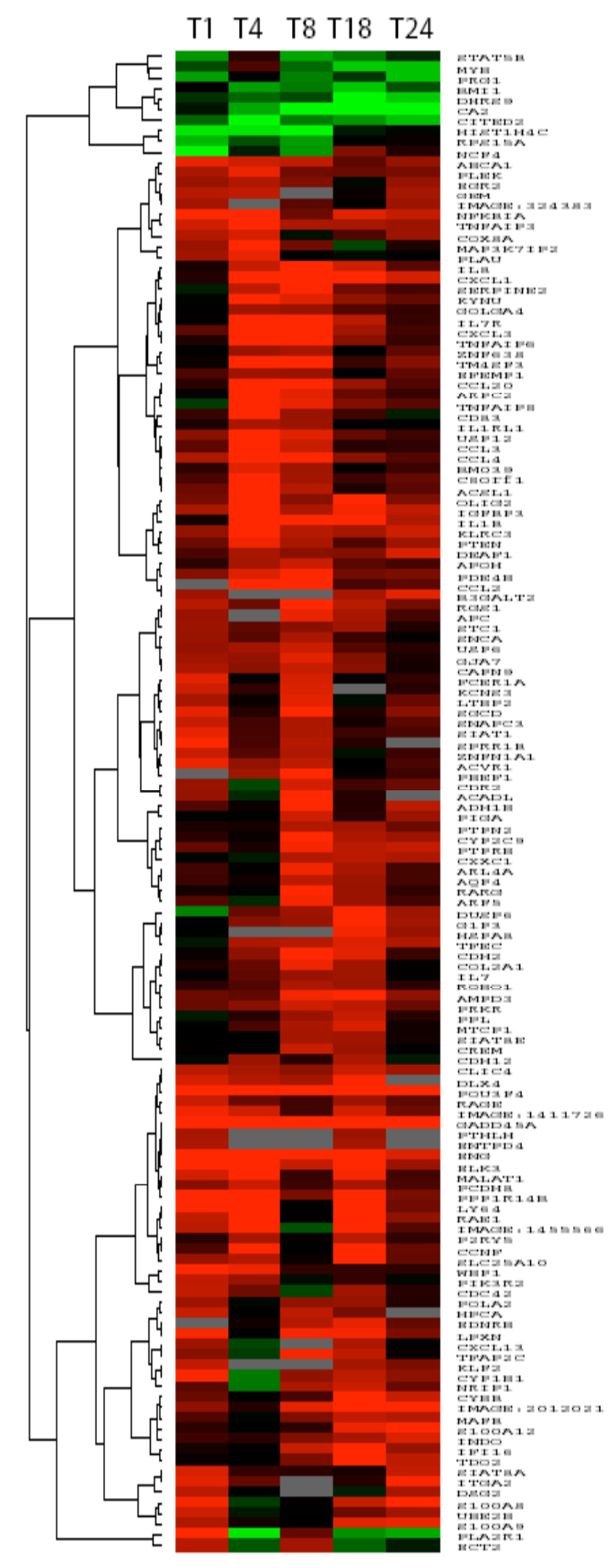

Fig. (1). H. cluster image of 142 genes with fluorescence ratios of $\geq 1.5$ or $\leq 0.667$ at any two time points compared to uninfected control. Lane 1 to 5 indicates the time course at T1-T24 after infection. The genes are ordered by similarity in their expression patterns across the time course. Color indicates relative expression intensity (red, increased; green, decreased; and grey, missing data). 
Table 2. 142 Genes Up-Regulated by $\geq 1.5$ Fold or Down-Regulated by $\leq$ 0.667 at More than One Time Points Post-Infection

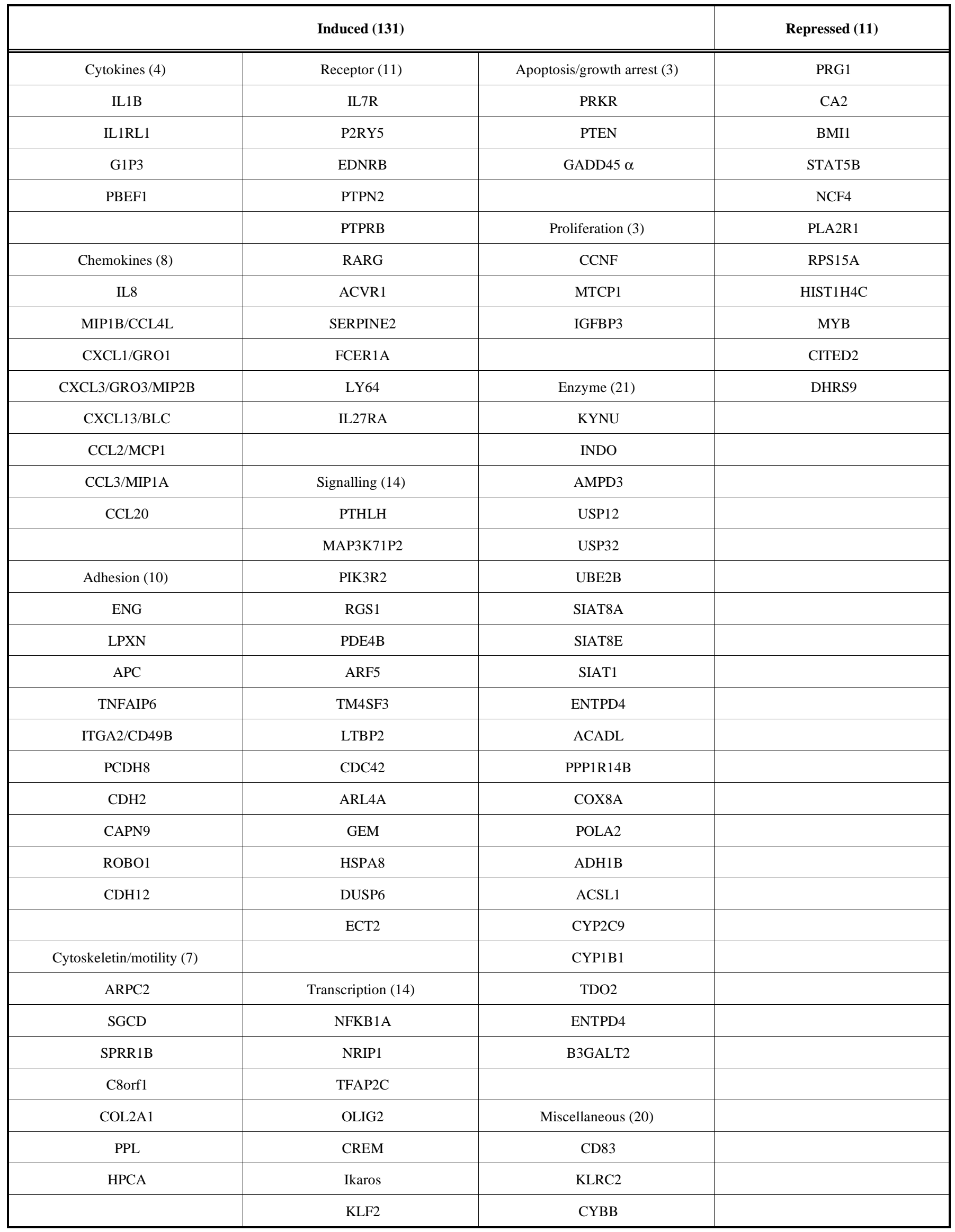


(Table 2). Contd.....

\begin{tabular}{|c|c|c|c|}
\hline \multicolumn{3}{|c|}{ Induced (131) } & \multirow[t]{2}{*}{ Repressed (11) } \\
\hline Transporter (6) & POU3F4 & DLX4 & \\
\hline AQP4 & IFI16 & EGR2 & \\
\hline $\mathrm{KCNS} 3$ & ELK3 & MAFB & \\
\hline SLC25A 10 & TFEC & CDR2 & \\
\hline CLIC4 & $\mathrm{CXXC1}$ & C6orf142 & \\
\hline $\mathrm{ABCA} 1$ & ZNF638 & DEAF1 & \\
\hline \multirow[t]{2}{*}{$\mathrm{APOH}$} & SNAPC3 & RAE1 & \\
\hline & & BM039 & \\
\hline Calcium ion binding (7) & Anti-apoptotic (3) & WBP1 & \\
\hline EFEMP1 & TNFAIP3 & MALAT1 & \\
\hline STC1 & TNFAIP8 & PLAU & \\
\hline PLEK & SNCA & GJA7 & \\
\hline S100A8 & & RAGE & \\
\hline S100A9 & & Unknown (image : 324383 ) & \\
\hline $\mathrm{S} 100 \mathrm{~A} 12$ & & Unknown (image : 1411726) & \\
\hline \multirow[t]{2}{*}{ DSG2 } & & Unknown (image : 1455566) & \\
\hline & & Unknown (image : 2012021) & \\
\hline
\end{tabular}

To explore the signature gene expression profiles over an infection period, we set the criteria that the change in expression level must be $\geq 1.5$ or $\leq 0.667$ fold at more than one time point. These criteria identified 131 up-regulated and 11 down-regulated genes that account for about $2 \%$ of the total 7,680 genes assessed. The differentially expressed genes were clustered hierarchically (Fig. 1). The pattern revealed remarkable changes in gene expression, including both upand down-regulation. Some genes were induced rapidly at T1 while others were induced at later times, most of the gene expressions returned to baseline level by T24. These results were presented in dendrograms in which the lengths of the branches are proportional to differences in gene expression of host at different time points.

These genes with a variety of biological functions represent a shared transcriptional response over the time course after infection. Their functions and biological processes involved were classified in Table 2. A total of $8.5 \%(12 / 142)$ of the induced genes functionally belong to cytokines (IL1B, IL1RL1, G1P3, and PBEF1) or chemokines (IL8, MIP1B, CXCL1, 3, 13, and CCL2, 3, 20), which are important for cell growth and inflammatory responses. Notably, several other subsets of genes were identified with functions related to adhesion, cytoskeleton/motility, and calcium ion binding. The responsive genes which function in apoptosis or antiapoptosis that were up-regulated included PRKR, PTEN, GADD45A, TNFAIP3, TNFAIP8, and SNCA. There were a large number of induced genes that were categorized as receptors or enzymes or have roles in signaling, transcription, and proliferation. We defined these 142 genes as the human monocyte signature genes that may reflect major signaling pathways or cascades stimulated upon the infection of $R$. prowazekii.

\section{Defining Common Outliers and Most Abundantly Up Regulated Transcripts}

Cluster analysis of expression profiles at these five time points indicated that those at $\mathrm{T} 4$ and $\mathrm{T} 8$, and $\mathrm{T} 18$ and $\mathrm{T} 24$ were more similar to each other by grouping in the same clusters, while that of $\mathrm{T} 1$ was the most dissimilar time point as illustrated by the dentrogram in Fig. (2A). Using the cutoff of $\geq 1.5$-fold, we found six genes (ENG, GADD45A, TNFAIP3, IGFBP3, POU3F4, and ELK3) were affected over the entire infection time course. Therefore they were designated as common outliers. Their expression patterns are shown in Fig. (2B). These genes were up-regulated at T1 and T4. The fold of increase declined at T8, and then up again at T18. All of them returned to 1.5-2.5 fold induction at T24.

We also searched for highly induced genes among signature genes that together with common outliers might serve as biomarkers for $R$. prowazekii infection. We set a criterion for this selection: the induction of expression should be 2.0 -fold or greater at more than one time point, or 3.0-fold or greater at a single time point. Twenty-two genes met this criterion (Table 3). Among them, six genes are cytokines or chemokines (IL1B, MIP1B, CXCL1, CXCL3, CCL2, and CCL20); three are transcription factors (NFKB1A, ELK3, POU3F4); and the others as receptors, or genes involved in signaling, cell cycle, anti-apoptosis, and adhesion, etc.

\section{IPA Pathway Analysis of Genes Affected at the Tran- scriptional Level After $R$. prowazekii Infection}

To further investigate the biological pathways and interactive networks of these genes affected by rickettsial infection, IPA was used. IPA distributes selected genes into networks defined by known interactions from scientific publications and then associates these networks with biological 
A Correlation Coefficient

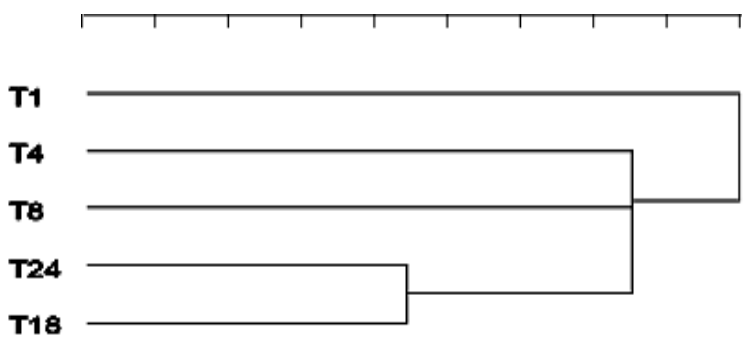

B

Gene expression profiles of common outliers

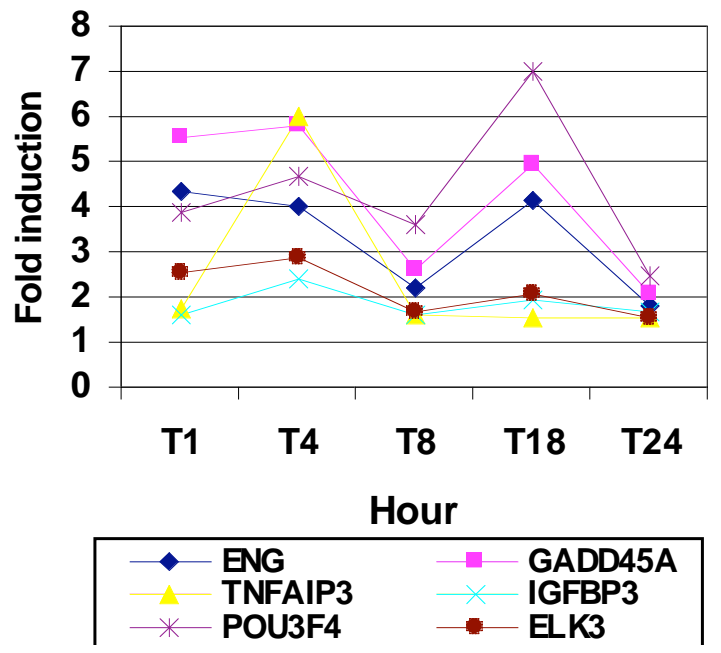

Fig. (2). The time course of gene response of THP-1 cells following $R$. prowazkeii infection. (A) Dendrogram comparing the correlation coefficient of host gene expression from T1-T24. (B) Graphical representation of the expression of common outliers through a time course.

Table 3. Twenty-Two THP-1 Genes Activated Post-Infection with $R$. prowazekii $(\geq 2.0$ in at Least Two Time Points or $\geq 3.0$ at a Single Time Point)

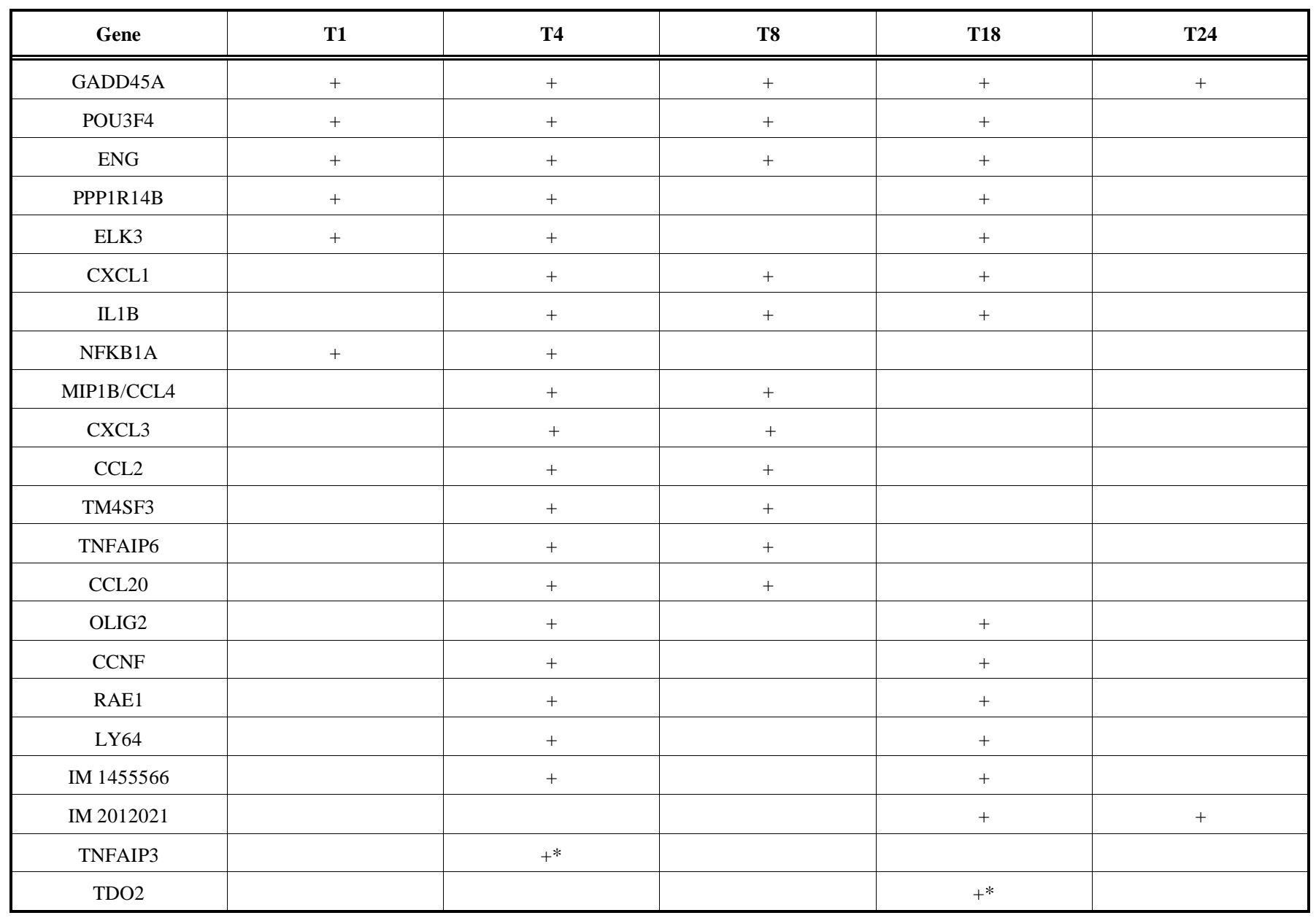


Table 4. Top 6 Networks Generated from IPA for Gene Expressions Affected by R. prowazekii Infection

\begin{tabular}{|c|c|c|c|}
\hline 1 & $\begin{array}{l}\text { ACSL1, ACVR1, AMPD3, BDKRB1, C9ORF26, CCBP2, CCL2, CCL3, CCL4, } \\
\text { CCL20, CD83, CITED2, COX8A, CREM, CXCL3, EGR2, GADD45A, HAS1, } \\
\text { IGFBP3, IL8, IL1B, IL1RL1, IRAK4, NFKBIA, PGDS, PLAU, PMP2, PTEN, } \\
\text { ROBO1, S100A8, S100A9, SCYE1, SLC25A4, TFAP2C, TNFAIP8 }\end{array}$ & 46 & $\begin{array}{l}\text { Cellular Movement, Cell-To-Cell } \\
\text { Signaling and Interaction, Viral Func- } \\
\text { tion }\end{array}$ \\
\hline 2 & $\begin{array}{l}\text { ACADL, ASS, CA2, CD82, CHST4, CLIC4, CXCL13, EDNRB, EIF2AK2, FABP1, } \\
\text { FABP5, FPR1, GADD45A, HNRPA1, HSD11B1, HSP90B1, HSPD1, IFNK, IL24, } \\
\text { INDO, ITGA4, ITGB7, KYNU, LPXN, LTBP2, NR1H3, NUTF2, PPARA, PPARD, } \\
\text { PTPN2, RAGE, RETN, RGS1, ST6GAL1, TNF }\end{array}$ & 21 & $\begin{array}{l}\text { Cardiovascular System Development } \\
\text { and Function, Cellular Movement, } \\
\text { Lipid Metabolism }\end{array}$ \\
\hline 3 & $\begin{array}{l}\text { ACVRL1, BRCA1, CAPN2, CAPN8, CAPN9, CAPN11, CDH2, CSH1, CTSD, } \\
\text { CYP1B1, DDX5, DSG2, DUSP6, EFEMP1, EGF, ELF3, ENG, ESR1, G1P3, } \\
\text { GADD45A, GJB1, GOLGA4, HIST1H4C, IRF6, IRS2, PIK3R2, PLSCR1, } \\
\text { PPP1R14B, PTGS1, SERPINB5, STC1, TERT, TGFB3, TGFBR2, VEGF }\end{array}$ & 19 & $\begin{array}{l}\text { Cancer, Cellular Movement, Drug } \\
\text { Metabolism }\end{array}$ \\
\hline 5 & $\begin{array}{l}\text { ABCA1, ARF5, BIRC2, CCL4, CDKN1A, CTSS, CYBA, CYBB, DIABLO, G1P2, } \\
\text { GADD45B, HGF, HNRPA2B1, IFNG, IL32, IRF2, KRT19, LDHA, NCF4, OCLN, } \\
\text { PDE4B, PIM1, PLA2G1B, PLA2R1, PLEK, PRTN3, PTPRB, RARG, SCYE1, } \\
\text { SLC2A1, SLC2A2, SPI1, ST8SIA1, TFEC, TNFAIP6 }\end{array}$ & 17 & $\begin{array}{l}\text { Cellular Growth and Proliferation, Cell } \\
\text { Death, Immune and Lymphatic System } \\
\text { Development and Function }\end{array}$ \\
\hline 6 & $\begin{array}{c}\text { APC, ARG1, ARPC2, ARPC5, CALM2, CDK2AP1, CDR2, CYP2C9, DEAF1, } \\
\text { DHRS9, E2F1, FCER1A, FCGR1A, FKBP5, G3BP, GBP2, GEM, HSP90B1, IER3, } \\
\text { KLF2, LDHA, MYC, NR3C1, POLA2, PPL, RHOB, ROCK1, ROCK2, RPS15A, } \\
\text { S100A6, SERPINF2, SGK, SPRR1B, TGFB1, TGTP }\end{array}$ & 15 & $\begin{array}{l}\text { Cancer, Cellular Growth and Prolifera- } \\
\text { tion, Endocrine System Disorders }\end{array}$ \\
\hline
\end{tabular}

*Bold genes were those identified by the microarray analysis (Bold only: up-regulated genes, bold with underline: down-regulated genes). Other genes were either not on the expression array or not significantly regulated. A score of $>3$ were considered statistically significant $(\mathrm{p}<0.001)$ by IPA.

pathways. IPA identified 10 statistically significant networks from 142 signature genes. The first six significant networks are shown in Table 4. Results showed that there are statistically significant interactions among these gene products that were modulated by $R$. prowazekii. These networks involve cellular movement, cell- to -cell signaling and interaction, lipid metabolism, cellular growth and proliferation, cell death, immune response, etc. Fig. (3) showed the top first network identified by IPA. Among this network, CCL2, IL1B, IL8, and NFKB1A seemed to be located at the center points to connect multiple points, suggesting their crucial roles in response to $R$. prowazekii.

\section{Confirmation of Responsive Genes by Real Time PCR}

From the lists of the most highly induced genes and the common outliers, the expressions of six genes were selected for further analysis by Taqman quantitative real-time reverse transcriptive PCR (RT-qPCR). The same preparation of RNA used in microarray experiment was used for qPCR with primers and probes specific for GADD45A, PPP1R14B, ENG, NFKB1A, IL1B, CXCL1, and TNFAIP3. All these genes showed an induction in different magnitude over the time course (Table 5). Generally speaking, the data from microarray screening and qPCR were in good agreement. The identification and confirmation of a core set of genes which were induced early in the rickettsial pathogenesis might be of value in the development of new strategies for early diagnosis.

\section{DISCUSSION}

The approach of global gene expression profiling on cDNA microarrays was employed and revealed a large number of genes differentially regulated. The results also suggested that the host response to rickettsial infection is a complicated process involving both induction and repression of groups of genes, with up-regulated genes the overwhelming majority. The genes induced by the infection were diverse in their functions as well. Overall, the alterations in the expression of these genes occurred in a time-dependent manner. Observing networks of differentially expressed genes during infection suggested that the co-expressed genes might also be co-regulated. The expression pattern in this study was compared with the features of eight other bacteria infections including Gram-negative, Gram-positive bacteria and mycobacteria [12]. This comparison revealed that about a quarter of 142 regulated genes caused by $R$. prowazekii infection were also reported in these infections. Majority of the genes identified in this study are not differentially expressed during other bacterial infections. It is reasonable to hypothesize that clusters of up- or down-regulated genes would constitute a signature pattern of expression, which might be specific for rickettsial infection (Table 2 ).

To establish the list of genes that responded most substantially to the pathogen $R$. prowazekii, a total of 22 host genes were identified (Table $\mathbf{3}$ ). Among them, the inductions of eight genes were detected at three or four time points (GADD45A, POU3F4, ENG, PPP1R14B, ELK3, CXCL1, 


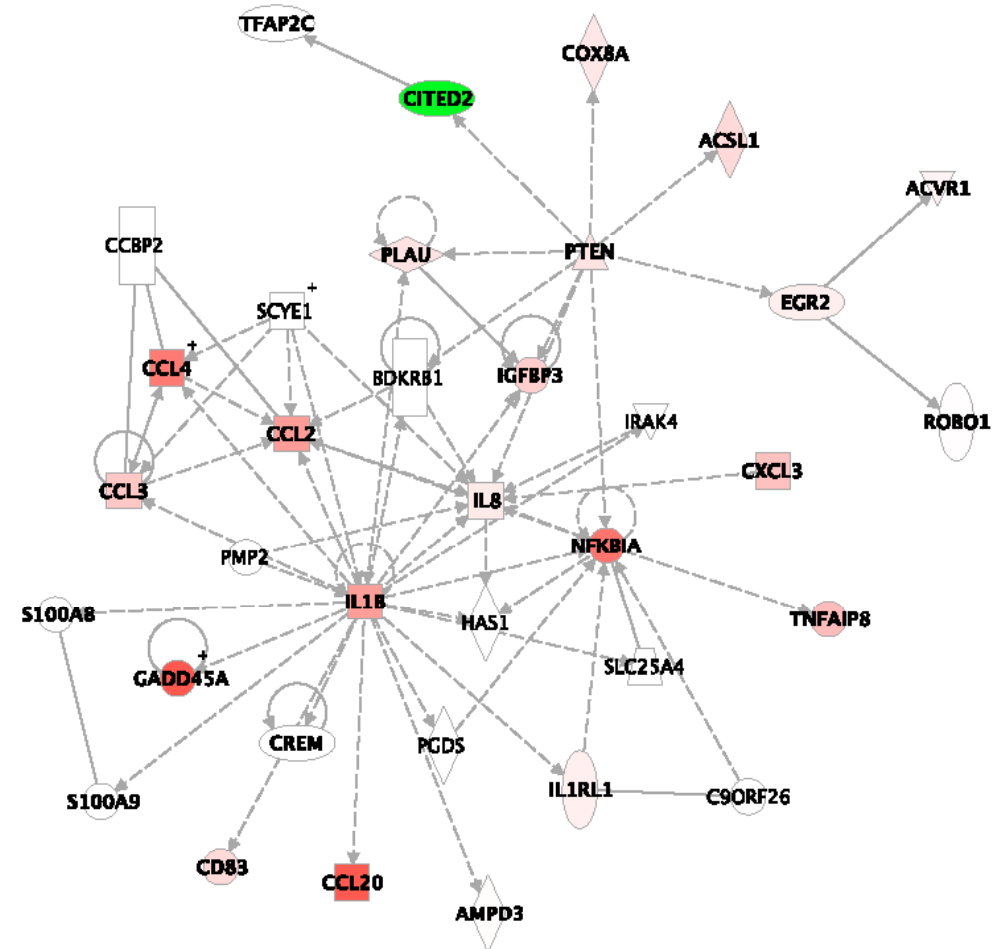

Fig. (3). A representative of networks of genes regulated after $R$. prowazekii infection. Twenty-five genes that were up-regulated (Red, the darker the higher induction) and one gene that was down-regulated (Green) in THP-1 cells were analyzed by Ingenuity Pathway Analysis (IPA) software. Other genes are those associated with the regulated genes.

Table 5. Quantitative Real-Time PCR for Selected Host Response Genes

\begin{tabular}{|c|c|c|c|c|c|c|c|c|c|c|}
\hline \multirow{2}{*}{$\begin{array}{c}\text { Gene } \\
18 \mathrm{~S} \mathrm{rRNA}^{\mathrm{d}}\end{array}$} & \multicolumn{10}{|c|}{$C_{T}{ }^{\mathrm{a}}(\mathrm{X} \pm \mathrm{SD})^{\mathrm{b}}$ and $^{\mathrm{c}}$ Fold Expression Change } \\
\hline & \multicolumn{2}{|c|}{ T1 } & \multicolumn{2}{|c|}{$\mathbf{T 4}$} & \multicolumn{2}{|c|}{ T8 } & \multicolumn{2}{|c|}{ T18 } & \multicolumn{2}{|c|}{$\mathbf{T 2 4}$} \\
\hline GADD45A & $\begin{array}{l}32.96 \pm 0.44 \\
33.40 \pm 0.20\end{array}$ & 1.88 & $\begin{array}{l}36.20 \pm 0.37 \\
32.62 \pm 0.63\end{array}$ & 6.25 & $\begin{array}{l}32.53 \pm 0.26 \\
32.43 \pm 0.20\end{array}$ & 3.43 & $\begin{array}{l}34.96 \pm 0.19 \\
36.05 \pm 0.40\end{array}$ & 4.63 & $\begin{array}{l}34.60 \pm 0.71 \\
34.14 \pm 0.58\end{array}$ & 2.17 \\
\hline ENG & $\begin{array}{l}33.64 \pm 1.21 \\
33.09 \pm 0.30\end{array}$ & 0.93 & $\begin{array}{l}37.38 \pm 0.98 \\
32.48 \pm 0.40\end{array}$ & 2.50 & $\begin{array}{l}31.56 \pm 0.02 \\
32.10 \pm 1.13\end{array}$ & 5.35 & $\begin{array}{l}33.56 \pm 0.35 \\
33.34 \pm 0.37\end{array}$ & 1.91 & $\begin{array}{l}31.50 \pm 0.38 \\
31.28 \pm 0.15\end{array}$ & 1.92 \\
\hline NFKB1A & $\begin{array}{l}26.15 \pm 0.07 \\
26.63 \pm 0.08\end{array}$ & 1.91 & $\begin{array}{l}27.38 \pm 0.22 \\
27.22 \pm 0.10\end{array}$ & 66.72 & $\begin{array}{l}26.11 \pm 0.15 \\
25.78 \pm 0.15\end{array}$ & 2.93 & $\begin{array}{l}26.47 \pm 0.08 \\
27.36 \pm 0.23\end{array}$ & 4.11 & $\begin{array}{l}25.45 \pm 0.17 \\
26.00 \pm 0.06\end{array}$ & 0.73 \\
\hline CXCL1 & $\begin{array}{l}32.05 \pm 0.34 \\
31.75 \pm 0.38\end{array}$ & 1.11 & $\begin{array}{l}33.22 \pm 0.36 \\
32.35 \pm 0.67\end{array}$ & 40.79 & $\begin{array}{l}30.83 \pm 0.38 \\
33.35 \pm 0.13\end{array}$ & 21.11 & $\begin{array}{l}30.32 \pm 0.26 \\
31.22 \pm 0.29\end{array}$ & 4.14 & $\begin{array}{l}28.68 \pm 0.39 \\
30.03 \pm 0.21\end{array}$ & 1.27 \\
\hline TNFAIP3 & $\begin{array}{l}36.31 \pm 0.61 \\
35.95 \pm 0.30\end{array}$ & 1.06 & $\begin{array}{l}37.18 \pm 0.15 \\
37.31 \pm 0.88\end{array}$ & 81.57 & $\begin{array}{l}36.38 \pm 0.07 \\
37.90 \pm 0.42\end{array}$ & 10.56 & $\begin{array}{l}37.87 \pm 0.19 \\
39.18 \pm 0.13\end{array}$ & 5.50 & $\begin{array}{l}35.60 \pm 0.81 \\
36.37 \pm 0.46\end{array}$ & 0.85 \\
\hline
\end{tabular}

${ }^{a} C_{T}$ represents the cycle number at which a significant increase in fluorescence signal above a threshold signal (horizontal zero line) can first be detected.

${ }^{b} \mathrm{X}$ and $\mathrm{SD}$, average and standard deviation values.

'Using the comparative $\left(\Delta \Delta C_{T}\right)$ method.

${ }^{\mathrm{d}}$ Internal control. 
IL1B, and NFKB1A) and others were seen at two time points. Six out of 22 genes belong to the category of cytokine or chemokine families, which are commonly seen in other infectious diseases. However, other genes are not commonly up-regulated due to microorganism infections and thus were not previously suspected of being transcriptionally regulated by rickettsial infection. Previous studies on these genes such as POU3F4 [13], PPP1R14B, TM4SF3 [14], OLIG2 [15], RAE1 [16], LY64 [17], and TDO2 [18] are very limited. For example, ENG, which plays a role in adhesion and receptor binding, was up-regulated in this study but was down-regulated in other infectious events including Gram positive and Gram negative bacteria [9]. POU3F4 (Brain-4) is a transcription factor mainly expressed in pancreatic cells [10] but its role in disease is unknown. In addition, six common outliers (ENG, GADD45A, TNFAIP3, IGFBP3, POU3F4, ELK3) that were up-regulated in the entire time course were identified. Five of them (ENG, POU3F4, TNFAIP3, GADD45A and ELK3) were also among the highly stimulated group.

Although we did not observe significant induction of IFN $\gamma$ which is usually up-regulated in rickettsial infection, we did notice the induction at T8 (1.2919). There was no increase at T1 (1.0042) and T24 (0.8695). Unfortunately, we excluded the IFN $\gamma$ data from $\mathrm{T} 4$ and $\mathrm{T} 18$ for analysis due to "bad spots". Therefore, the status of IFN $\gamma$ in this study is not fully clear. We also saw increases of IFN $\gamma$ receptor 2 at T1 (1.3512) and T4 (1.9655) in this study.

By using IPA, the interactive networks of genes were constructed. IPA identified statistically significant genes that participate in the multiple pathways with mutual interactions. Further functional analysis of these associated networks and pathways as well as dose response and protein level may provide additional insight into the macrophage defense response against $R$. prowazekii infection.

\section{CONCLUSION}

Our findings have uncovered the molecular features of host responses to $R$. prowazekii infection at genome-wide level. In addition, this study may pave the road for further studies of interaction between rickettsia and host.

\section{ACKNOWLEDGMENTS}

This work was supported by the Naval Medical Research and Development Command, Work Unit 6000.RAD1.J.
A0310. The views expressed in this article are those of the author and do not necessarily reflect the official policy or position of the Department of the Navy, Department of Defense, nor the U.S. Government. We would like to thank Lieutenant Paul Graf for comments and suggestions of this manuscript.

\section{REFERENCES}

[1] Gross, L. Proc. Natl. Acad. Sci. USA, 1996, 93,10539-40.

[2] Kelly, D.J.; Richards, A.L.; Temenak, J.; Strickman, D.; Dasch, G.A. Clin. Infect. Dis., 2002, 34, S145-S69.

[3] Knodler, L.A.; Celli, J.; Finlay, B.B. Pathogenic trickery: deception of host cell processes. Nat. Rev. Mol. Cell Biol., 2001, 2, 578-88.

[4] Andersson, S.G.; Zomorodipour, A.; Andersson, J.O.; SicheritzPonten, T.; Alsmark, U.C.M.; Podowski, R.M.; Naslung, A.K.; Eriksson, A.S.; Winkler, H.H.; Kurland, C.G. Nature, 1998, 396,130-40.

[5] Ge, H.; Chuang, Y-YE.; Zhao, S.; Tong, M. ; Tsai, M-H.; Temenak, J.J.; Richards, A.L.; Ching, W-M. J. Bacteriol., 2004, 186, 556-65.

[6] Eremeeva, M.E.; Dasch, G.A.; Silver, D.J. In Bacterial invasion into eukaryotic cells; Oelschlaeger, T.A. and Hacker J, Ed.; Lkuwer Academic/Plenum Publishers, New York, 2000; Vol. 33, pp. 479516.

[7] Teysseire, N.; Boudie,r J-A.; Raoult, D. Infect. Immun., 1995, 63, 366-74.

[8] Gouin, E.; C. Egile, P.; Dehoux, V.; Villiers, J.; Adams, F.; Gertler, R.; Li, Cossart, P. Nature, 2004, 427, 457-61.

[9] Eisen, M.B.; Spellman, P.T.; Brown, P.O.; Botstein, D. Proc. Natl. Acad. Sci. USA, 1998, 95, 14863-8.

[10] Lu, X.; da la Pena, L.; Barker, C.; Camphausen, K.; Tofilon, P.J. Cancer Res., 2006, 66, 1052-61.

[11] Raponi, M.; Belly, R.T.; Karp, J.E.; Lancet, .JE.; Atkins, D.; Wang, Y. BMC Cancer, 2004, 4, 56-66.

[12] Nau, G.J.; Richmond, J.F.L.; Schlesinger, A.; Jennings, E.G.; Lander, E.S.; Young, R.A. Proc. Natl. Acad. Sci. USA, 2002, 99, 1503-8.

[13] Heller, R.S.; Stoffers, D.A.; Liu, A.; Schedl, A.; Crenshaw, E.B.; Madsen, O.D.; Serup, P. Dev. Biol., 2004, 268, 123-34.

[14] Lo, D.; Tynan, W.; Dickerson, J.; Scharf, M.; Cooper, J.; Byrne, D.; Brayden, D.; Higgins, L.; Evans, C.; O’Mahony, D.J. Int. Immunol., 2004, 16, 91-9.

[15] Furusho, M.; Ono, K.; Takebayashi, H.; Masahira, N.; Kagawa, T.; Ikeda, K.; Ikenaka, K. Dev. Biol., 2006, 293, 348-57.

[16] Babu, J.R.; Jeganathan, K.B.; Baker, D.J.; Wu, X.; Kang-Decker, N.; van Deursen, J.M. J. Cell Biol., 2003, 160, 341-53.

[17] Yazawa, N.; Fujimoto, M.; Sato, S.; Miyake, K.; Asano, N.; Nagai, Y.; Takeuchi, O.; Takeda, K.; Okochi, H.; Akira, S.; Tedder, T.F.; Tamaki, K. Blood, 2003, 102, 1374-80.

[18] Vasiliev, G.V.; Merkulov, V.M.; Kobzev, V.F.; Merkulova, T.I.; Ponomarenko, M.P.; Kolchanov, N.A. FEBS lett., 1999, 462, 85-8.

Received: December 31, 2007

Accepted: April 15, 2008

(C) Ge et al.; Licensee Bentham Open.

This is an open access article distributed under the terms of the Creative Commons Attribution License (http://creativecommons.org/license/by/2.5/), which permits unrestrictive use, distribution, and reproduction in any medium, provided the original work is properly cited. 